\title{
Meat Consumption, Related Nutrients, Obesity and Risk of Prostate Cancer: a Case-Control Study in Uruguay
}

\author{
Eduardo De Stefani ${ }^{*}$, Paolo Boffetta ${ }^{2}$,Alvaro L Ronco ${ }^{3,4}$,Hugo Deneo-Pellegrini ${ }^{1}$
}

\begin{abstract}
Background: In order to determine the role of meat consumption and related nutrients in the etiology of prostate cancer we conducted a case-control study among Uruguayan men in the time period 1998-2007. Results: The study included 464 cases and 472 controls, frequency matched for age and residence. Both series were drawn from the four major public hospitals in Montevideo. Unconditional logistic regression was used to estimate odds ratios (ORs) and $95 \%$ confidence intervals (95\% CI) of prostate cancer by quartiles of meat intake and related nutrients. The highest vs. the lowest quartile of intake of total meat $(\mathrm{OR}=5.19,95 \% \mathrm{CI}$ 3.46-7.81), red meat $(O R=4.64,95 \%$ CI 3.10-6.95), and processed meat $(O R=1.78,95 \%$ CI 1.22-2.59) were associated with increased risk of prostate cancer. Meat nutrients were directly associated with the risk of prostate cancer (OR for cholesterol 5.61, $95 \%$ CI 3.75-8.50). Moreover, both total meat and red meat displayed higher risks among obese patients. Conclusions: This study suggests that total and red meat and meat nutrients may play a role in the etiology of prostate cancer in Uruguay.
\end{abstract}

Keywords: Prostate cancer - red meat - processed meat - poultry - fish

Asian Pac J Cancer Prev, 17 (4), 1937-1945

\section{Introduction}

Prostate cancer is the leading malignancy in Uruguayan men with an age-standardized rate of 55.7 cases per 100,000 persons (Barrios et al., 2010). Thus, prostate cancer is a major public health problem in this country.

The etiology of prostate cancer remains a challenge for researchers. In the monograph of World Cancer Research Fund/American Institute for Cancer Research, total fat and saturated fat were considered possible causes of this disease (1997). More recently, a new monograph of the World Cancer Research Fund/American Institute for Cancer Research considered that diets rich in calcium are probable factors of prostate cancer (2007). Also, processed meat, milk and dairy products were considered as suggestive factors which increased the risk of this malignancy (World Cancer Research Fund/American Institute for Cancer Research, 2007).

Several publications (Deneo-Pellegrini et al, 1999; Cross et al., 2005; Rodríguez et al., 2006; Koutros et al., 2008; Aune et al. 2009; Sinha et al., 2009; Alexander et al., 2010; Richman et al., 2011; Punnen et al., 2011), considered that meat consumption could be a major risk factor for this malignancy and obesity may play an important role in prostate carcinogenesis (Giovannucci et al., 2003).

According to the FAO Uruguay has the highest beef consumption per head in the world, but with a population of only 3.3 million people, it also exports around $75 \%$ of its total beef production (FAO, 2001; Matos \& Brandani., 2002). For these reasons we decided to conduct a casecontrol study on the role of meat consumption and related nutrients in the etiology of prostate cancer.

\section{Materials and Methods}

\section{Selection of cases}

In the time period 1998-2007 all newly diagnosed and microscopically confirmed cases of prostate cancer were considered eligible for this study. A total of 475 cases were identified in the four major public hospitals located in Montevideo. Eleven patients refused the interview, leaving a final total of 464 cases (response rate $97.6 \%$ ). The stage distribution was as follows: localized, $25 \%$; regional, $72 \%$; and diseminated, $3 \%$. There were no cases with latent carcinomas, and therefore, this series is representative of a series of mainly advanced prostate tumors. The stage distribution of our series was compared with the figures drawn from the National Cancer Registry. According to this source, $70 \%$ of prostate cancers were locally advanced (regional) or disseminated at the time of the diagnosis. These figures reflect the fact that there are no mass screening programmes for prostate cancer in Uruguay. 


\section{Selection of controls}

In the same time period (1998-2007) and in the same hospitals (four major public health hospitals of Montevideo), patients with non-neoplastic diseases not related to tobacco smoking, alcohol drinking and without recent changes in their diets were considered as eligible for the study. We identified 1,689 patients and 49 of them refused the interview, leaving a final total of 1,640 potential controls (response rate $97.1 \%$ ). We randomly selected 472 persons from the potential controls and frequency matched to the cases on categorical age (10year intervals) and residence. These controls presented the following diseases: eye disorders (137 patients, 29.0 $\%)$, abdominal hernia $(117,24.8 \%)$, fractures $(48,10.2$ $\%)$, diseases of the skin $(30,6.4 \%)$, urinary stones $(29$, $6.1 \%$ ), injuries $(27,5.7 \%)$, venous disorders $(26,5.5 \%)$, acute appendicitis $(18,3.8 \%)$, hydatid cyst $(17,3.6 \%)$, blood disorders $(15,3.2 \%)$, and peritonitis $(8,1.7 \%)$.

\section{Interviews and questionnaire}

Both cases and controls were administered a structured questionnaire by two trained social workers. All the interviews were conducted in the hospitals shortly after admittance. The questionnaire included the following sections: socio-demographics (age, residence, education, income, identity number), a complete history of occupation including the four last jobs and their duration, family history of prostate cancer in the firstdegree relatives (father, brothers), self-reported height and weight 5 years before the date of the interview, a complete history of smoking (age of start, age of quit, number of cigarettes smoked per day, type of cigarette, type of tobacco, inhalation practices), a complete history of alcohol drinking (age of start, age of quit, number of glasses drunk per day, type of alcoholic beverage), a complete history of nonalcoholic beverages (coffee, tea, mate), and a food frequency questionnaire (FFQ), which queried the usual intake of 64 food items one year prior to diagnosis. This FFQ allowed the calculation of total energy intake and represented the usual diet of the Uruguayan population. Although the FFQ was not validated, it was tested for reproducibility. The correlation coefficients between the two diet assessments were 0.77 for red meat, 0.60 for white meat, 0.55 for processed meat, and 0.67 for total meat (Ronco et al., 2006).

\section{Definitions of meats and nutrients}

We calculated the following items: (1) total meat (red meat, processed meat, poultry, fish, liver), (2) red meat (beef, lamb), (3) processed meat (bacon, sausage, mortadella, salami, saucisson, hot dog, ham, salted meat), (4) total white meat (poultry, fish), (5) poultry, (6) fish, and (7) liver. Nutrient intake was assessed using a Spanish database (Mazzei et al., 1995). The following nutrients were estimated: animal protein, saturated fat, monounsaturated fat, polyunsaturated fat, linoleic acid, $\alpha$-linolenic acid, cholesterol, vitamin B12, iron, phosphorus, and nitrosodimethylamine (NDMA).

All the study variables (meat and nutrients) were logtransformed and energy-adjusted by the residuals method of Willett and Stampfer (Willett, 1998). Meat intake was categorized in quartiles according to the distribution of the cases and controls.

Table 1. Distribution of Cases and Controls by Socio-demographics and Related Variables

\begin{tabular}{|c|c|c|c|c|c|c|}
\hline \multirow{2}{*}{ Variable } & \multirow{2}{*}{ Category } & \multicolumn{2}{|c|}{ Cases } & \multicolumn{2}{|c|}{ Controls } & \multirow{2}{*}{ Global p-value } \\
\hline & & $\mathrm{N}^{\circ}$ & $\%$ & $\mathrm{~N}^{\circ}$ & $\%$ & \\
\hline \multirow[t]{5}{*}{ Age (years) } & $40-49$ & 3 & 0.7 & 4 & 0.8 & \multirow{5}{*}{0.92} \\
\hline & $50-59$ & 37 & 8.0 & 38 & 8.0 & \\
\hline & $60-69$ & 149 & 32.0 & 163 & 34.6 & \\
\hline & $70-79$ & 226 & 48.7 & 217 & 46.0 & \\
\hline & $80-89$ & 49 & 10.6 & 50 & 10.6 & \\
\hline \multirow[t]{2}{*}{ Residence } & Montevideo & 230 & 49.6 & 228 & 48.3 & \multirow{2}{*}{0.70} \\
\hline & Other counties & 234 & 50.4 & 244 & 51.7 & \\
\hline \multirow[t]{3}{*}{ Education (yrs) } & $0-2$ & 133 & 28.7 & 128 & 27.1 & \multirow{3}{*}{0.72} \\
\hline & $3-6$ & 179 & 38.6 & 178 & 37.7 & \\
\hline & $7+$ & 152 & 32.7 & 166 & 35.2 & \\
\hline \multirow[t]{3}{*}{ Income (US dollars) } & $<146$ & 157 & 33.8 & 171 & 36.2 & \multirow{3}{*}{0.87} \\
\hline & $147+$ & 175 & 37.8 & 186 & 39.4 & \\
\hline & Missing & 132 & 28.4 & 115 & 24.4 & \\
\hline \multirow[t]{4}{*}{ Hospital } & Cancer & 118 & 25.4 & 122 & 26.1 & \multirow{4}{*}{0.99} \\
\hline & Pasteur & 115 & 24.8 & 116 & 24.6 & \\
\hline & Clínicas & 143 & 30.8 & 145 & 30.8 & \\
\hline & Maciel & 88 & 19.0 & 87 & 18.5 & \\
\hline \multirow[t]{2}{*}{ Family history prostate cancer } & No & 451 & 97.2 & 467 & 98.9 & \multirow{2}{*}{0.05} \\
\hline & Yes & 13 & 2.8 & 5 & 1.1 & \\
\hline \multirow[t]{2}{*}{ Body Mass Index $\left(\mathrm{kg} / \mathrm{m}^{2}\right)$} & $<23.2$ & 94 & 20.3 & 141 & 29.8 & \multirow[t]{2}{*}{$<0.0001$} \\
\hline & $23.3-25.2$ & 104 & 22.4 & 132 & 28.0 & \\
\hline
\end{tabular}


Statistical analysis

Relative risks, approximated by the odds ratios, were estimated by multiple unconditional logistic regression (Rothman et al., 2008). Odds ratios of meat and/or nutrients were estimated fitting the following model: age (continuous), residence (ordinal), education (categorical, 3 strata), family history of prostate cancer (ordinal), body mass index (categorical, 4 strata), and total energy intake (continuous). Odds ratios for each histologic category of controls were fitted by multinomial (polytomous) regression (Rothman et al., 2008). Heterogeneity was estimated by the likelihood ratio test, and p-value for trend was calculated as entering categorical variables as continuous in the model. Goodness of fit of each model was calculated by the Hosmer-Lemeshow statistic (Hosmer \& Lemeshow., 1980). A two-tailed p-value of $<0.05$ was considered to be statistical significant. All statistical analyses were carried out using Stata version
13.1 (Stata, 2013). The study was approved by the Directors of the public health hospitals in Montevideo.

\section{Results}

The distribution of cases and controls by sociodemographic variables and selected risk factors is shown in Table 1. As expected from the matched design, age and residence were rather similar in cases and controls. Cases were less educated that controls but the difference was not significant (global p-value $=0.72$ ). On the other hand, cases and controls showed similar monthly income (global p-value $=0.87$ ). The distribution of cases and controls by hospital was similar for both groups of participants (global p-value $=0.99$ ) and family history of prostate cancer in first-degree relatives was more frequent among cases compared with controls (global p-value $=0.05$ ). Finally, cases were significantly more obese than controls (global p-value $<0.0001)$.

Table 2. Odds Ratios of Advanced Prostate Cancer for Meat Intake ${ }^{a}$

\begin{tabular}{|c|c|c|c|c|}
\hline Type of meat & Grams/day & Cases/Controls & OR & $95 \% \mathrm{CI}$ \\
\hline \multirow[t]{5}{*}{ Total meat } & $<95.5$ & $69 / 165$ & 1.0 & reference \\
\hline & $95.6-151.5$ & $108 / 126$ & 2.05 & $1.39-3.02$ \\
\hline & 151.6-176.7 & $123 / 111$ & 2.54 & $1.72-3.75$ \\
\hline & $176.8+$ & $164 / 70$ & 5.19 & $3.46-7.81$ \\
\hline & & $\mathrm{p}$-value for trend & \multicolumn{2}{|c|}{$<0.0001$} \\
\hline \multirow[t]{5}{*}{ Red meat } & $<74.8$ & $76 / 158$ & 1.0 & reference \\
\hline & 74.9-134.2 & $102 / 132$ & 1.65 & $1.12-2.42$ \\
\hline & 134.3-154.9 & $124 / 110$ & 2.33 & $1.58-3.43$ \\
\hline & $155.0+$ & $162 / 72$ & 4.64 & $3.10-6.95$ \\
\hline & & $\mathrm{p}$-value for trend & \multicolumn{2}{|c|}{$<0.0001$} \\
\hline \multirow[t]{5}{*}{ Processed meat } & $<9.0$ & $96 / 138$ & 1.0 & reference \\
\hline & $9.1-20.0$ & $126 / 108$ & 1.64 & $1.13-2.39$ \\
\hline & $20.1-36.3$ & $110 / 124$ & 1.25 & $0.86-1.82$ \\
\hline & $36.4+$ & $132 / 102$ & 1.78 & $1.22-2.59$ \\
\hline & & $\mathrm{p}$-value for trend & \multicolumn{2}{|c|}{0.017} \\
\hline \multirow[t]{5}{*}{ White meat } & $<5.9$ & $101 / 133$ & 1.0 & reference \\
\hline & $6.0-14.6$ & $122 / 113$ & 1.52 & $1.04-2.21$ \\
\hline & $14.7-23.2$ & $137 / 97$ & 1.96 & $1.33-2.84$ \\
\hline & $23.3+$ & $104 / 129$ & 1.06 & $0.72-1.56$ \\
\hline & & $\mathrm{p}$-value for trend & \multicolumn{2}{|c|}{0.51} \\
\hline \multirow[t]{5}{*}{ Poultry } & $<2.6$ & $98 / 136$ & 1.0 & reference \\
\hline & $2.7-11.2$ & $120 / 115$ & 1.44 & $0.99-2.10$ \\
\hline & $11.3-11.6$ & $133 / 100$ & 2.01 & $1.37-2.34$ \\
\hline & $11.7+$ & $113 / 121$ & 1.31 & $0.89-1.91$ \\
\hline & & $\mathrm{p}$-value for trend & \multicolumn{2}{|c|}{0.07} \\
\hline \multirow[t]{5}{*}{ Fish } & 0 & $113 / 121$ & 1.0 & reference \\
\hline & $1.0-2.8$ & $112 / 122$ & 1.00 & $0.69-1.46$ \\
\hline & $2.9-12.0$ & $119 / 115$ & 1.10 & $0.76-1.61$ \\
\hline & $12.1+$ & $120 / 114$ & 1.09 & $0.74-1.60$ \\
\hline & & $\mathrm{p}$-value for trend & \multicolumn{2}{|c|}{0.57} \\
\hline \multirow[t]{5}{*}{ Liver } & 0 & $118 / 116$ & 1.0 & reference \\
\hline & $0.1-1.0$ & $113 / 121$ & 1.03 & $0.67-1.59$ \\
\hline & $1.1-1.2$ & $123 / 111$ & 1.37 & $0.77-2.44$ \\
\hline & $1.3+$ & $110 / 124$ & 0.94 & $0.63-1.42$ \\
\hline & & $\mathrm{p}$-value for trend & \multicolumn{2}{|c|}{0.7} \\
\hline
\end{tabular}

${ }^{a}$ Multivariate adjusted for age, residence, education, body mass index, and total energy intake 
Table 3. Odds Ratios of Advanced Prostate Cancer for Meat Consumption Stratified By Body Mass Index ${ }^{a}$

\begin{tabular}{|c|c|c|c|c|c|}
\hline \multicolumn{6}{|c|}{ Body Mass Index } \\
\hline \multirow{2}{*}{ Type of meat } & \multicolumn{2}{|c|}{ Low BMI } & \multicolumn{2}{|c|}{ High BMI } & \multirow{2}{*}{ p-value heterogeneity } \\
\hline & OR & $95 \%$ CI & OR & $95 \%$ CI & \\
\hline \multirow[t]{5}{*}{ Total meat } & 1.0 & reference & 1.0 & reference & \\
\hline & 2.31 & $1.37-3.91$ & 1.93 & $1.08-3.43$ & \\
\hline & 2.35 & $1.38-4.01$ & 3.01 & $1.68-5.89$ & \\
\hline & 3.42 & $1.94-6.00$ & 8.97 & $4.86-16.5$ & \\
\hline & \multicolumn{2}{|c|}{$<0.0001^{\mathrm{c}}$} & \multicolumn{2}{|c|}{$<0.0001$} & 0.04 \\
\hline \multirow[t]{5}{*}{ Red meat } & 1.0 & reference & 1.0 & reference & \\
\hline & 1.38 & $0.83-2.30$ & 1.93 & $1.08-3.45$ & \\
\hline & 1.53 & $0.89-2.62$ & 3.69 & $2.09-6.53$ & \\
\hline & 2.88 & $1.66-4.99$ & 7.60 & $4.17-13.8$ & \\
\hline & \multicolumn{2}{|c|}{0.0003} & \multicolumn{2}{|c|}{$<0.0001$} & 0.04 \\
\hline \multirow[t]{5}{*}{ Processed meat } & 1.0 & reference & 1.0 & reference & \\
\hline & 1.41 & $0.83-2.38$ & 1.89 & $1.11-3.20$ & \\
\hline & 0.96 & $0.57-1.61$ & 1.70 & $0.99-2.92$ & \\
\hline & 1.51 & $0.90-2.55$ & 2.18 & $1.28-3.73$ & \\
\hline & \multicolumn{2}{|c|}{0.31} & \multicolumn{2}{|c|}{0.01} & 0.48 \\
\hline \multirow[t]{5}{*}{ Total white meat } & 1.0 & reference & 1.0 & reference & \\
\hline & 1.93 & $1.13-3.32$ & 1.24 & $0.73-2.11$ & \\
\hline & 1.98 & $1.12-3.47$ & 2.05 & $1.20-3.51$ & \\
\hline & 1.50 & $0.85-2.65$ & 0.89 & $0.53-1.50$ & \\
\hline & \multicolumn{2}{|c|}{0.23} & \multicolumn{2}{|c|}{0.86} & 0.32 \\
\hline \multirow[t]{5}{*}{ Poultry } & 1.0 & reference & 1.0 & reference & \\
\hline & 1.73 & $0.99-3.00$ & 1.20 & $0.72-2.01$ & \\
\hline & 2.37 & $1.38-4.09$ & 1.66 & $0.96-2.84$ & \\
\hline & 1.99 & $1.13-3.35$ & 0.96 & $0.57-1.63$ & \\
\hline & \multicolumn{2}{|c|}{0.01} & \multicolumn{2}{|c|}{0.8} & 0.29 \\
\hline \multirow[t]{5}{*}{ Fish } & 1.0 & reference & 1.0 & reference & \\
\hline & 1.17 & $0.69-1.99$ & 0.75 & $0.43-1.28$ & \\
\hline & 1.08 & $0.63-1.85$ & 1.02 & $0.60-1.75$ & \\
\hline & 1.41 & $0.83-2.39$ & 0.88 & $0.49-1.55$ & \\
\hline & \multicolumn{2}{|c|}{0.25} & \multicolumn{2}{|c|}{0.99} & 0.18 \\
\hline \multirow[t]{5}{*}{ Liver } & 1.0 & reference & 1.0 & reference & \\
\hline & 0.92 & $0.50-1.71$ & 1.21 & $0.65-2.25$ & \\
\hline & 2.60 & $1.13-5.78$ & 0.77 & $0.34-1.74$ & \\
\hline & 1.13 & $0.64-1.98$ & 0.81 & $0.45-1.45$ & \\
\hline & \multicolumn{2}{|c|}{0.49} & \multicolumn{2}{|c|}{0.27} & 0.08 \\
\hline
\end{tabular}

${ }^{a}$ Multivariate odds ratios adjusted for age, residence, education, and total energy intake. Cutpoints: low BMI $<=25.29$, high BMI $>=25.30$.

As shown in Table 2, the multivariate adjusted ORs comparing the highest vs. the lowest quartile of intake was 5.19 (95\% CI 3.46-7.81, p-value for trend < 0.0001) for total meat intake, 4.64 (95\% CI 3.10-6.95) for red meat, 1.78 (95\% CI 1.22-2.59) for processed meat, $1.31(95 \%$ CI $0.89-1.84)$ for poultry, and 1.09 (95 \% CI 0.74-1.60) for fish. Although intake of total white meat did not show a significant dose-response, those ORs corresponding to the second and third quartiles were significantly associated with the risk of the disease.

Odds ratios of prostate cancer for meat intake, stratified by body mass index, are shown in Table 3 . The effects of total meat (OR 8.97, $95 \%$ CI 4.86-16.5) and red meat (OR 7.60, $95 \%$ CI 4.17-13.8) were stronger among obese patients, and the likelihood ratio test was significant in both types of meat (p-value $=0.04)$.
The effect of meat nutrients are shown in Table 4. All the nutrients increased the risk of prostate cancer with a significant dose-response. The strongest association was observed for cholesterol (OR 5.61, $95 \%$ CI 3.75$8.50, \mathrm{p}$-value for trend $<0.0001)$, whereas the weakest associations were found for NDMA (OR 1.68, 95\% CI $1.14-2.48, \mathrm{p}$-value for trend $=0.004)$ and total iron $(\mathrm{OR}$ $1.55,95 \%$ CI 1.06-2.25, p-value $=0.02$ ).

Odds ratios of prostate cancer for meat nutrients, stratified by body mass index, are shown in Table 5 . ORs of animal protein, monounsaturated fat, cholesterol, vitamin B12, and NDMA were significantly associated with obese patients.

\section{Discussion}


Table 4. Odds Ratios of Advanced Prostate Cancer for Meat Nutrients a

\begin{tabular}{|c|c|c|c|c|}
\hline Meat nutrients & Category & Cases/Controls & OR & $95 \% \mathrm{CI}$ \\
\hline \multirow[t]{5}{*}{ Animal protein ${ }^{\mathrm{b}}$} & $<34.9$ & $77 / 157$ & 1.0 & reference \\
\hline & $35.0-45.5$ & $101 / 133$ & 1.65 & $1.12-2.43$ \\
\hline & $45.6-57.8$ & $131 / 103$ & 2.57 & $1.74-3.78$ \\
\hline & $57.9+$ & $155 / 79$ & 4.08 & $2.74-6.07$ \\
\hline & & $\mathrm{p}$-value for trend & \multicolumn{2}{|c|}{$<0.0001$} \\
\hline \multirow[t]{5}{*}{ Saturated fat ${ }^{\mathrm{b}}$} & $<26.7$ & $71 / 163$ & 1.0 & reference \\
\hline & $26.8-36.4$ & $110 / 125$ & 2.00 & $1.36-2.95$ \\
\hline & $36.5-47.4$ & $135 / 98$ & 3.34 & $2.25-4.95$ \\
\hline & $47.5+$ & $148 / 86$ & 4.24 & $2.82-6.38$ \\
\hline & & $\mathrm{p}$-value for trend & \multicolumn{2}{|c|}{$<0.0001$} \\
\hline \multirow[t]{5}{*}{ Monounsaturated fat ${ }^{\mathrm{b}}$} & $<24.5$ & $72 / 162$ & 1.0 & reference \\
\hline & $24.6-34.2$ & $106 / 128$ & 1.87 & $1.27-2.75$ \\
\hline & $34.3-43.9$ & $132 / 102$ & 2.95 & $1.99-4.36$ \\
\hline & $44.0+$ & $154 / 80$ & 4.52 & $3.00-6.79$ \\
\hline & & $\mathrm{p}$-value for trend & \multicolumn{2}{|c|}{$<0.0001$} \\
\hline \multirow[t]{5}{*}{ Polyunsaturated fat ${ }^{\mathrm{b}}$} & $<4.9$ & $72 / 162$ & 1.0 & reference \\
\hline & $5.0-6.2$ & $126 / 108$ & 2.62 & $1.78-3.86$ \\
\hline & $6.3-8.0$ & $137 / 97$ & 3.17 & $2.15-4.69$ \\
\hline & $8.1+$ & $129 / 105$ & 2.89 & $1.94-4.20$ \\
\hline & & $\mathrm{p}$-value for trend & \multicolumn{2}{|c|}{$<0.0001$} \\
\hline \multirow[t]{5}{*}{ Linoleic acid ${ }^{b}$} & $<3.9$ & $94 / 140$ & 1.0 & reference \\
\hline & $4.0-5.1$ & $125 / 109$ & 1.68 & $1.15-2.44$ \\
\hline & $5.2-6.6$ & $116 / 118$ & 1.48 & $1.02-2.15$ \\
\hline & $6.7+$ & $129 / 105$ & 1.87 & $1.28-2.73$ \\
\hline & & $\mathrm{p}$-value for trend & \multicolumn{2}{|c|}{0.004} \\
\hline \multirow[t]{5}{*}{$\alpha$-Linolenic acid ${ }^{b}$} & $<0.7$ & $70 / 164$ & 1.0 & reference \\
\hline & $0.8-1.0$ & $110 / 124$ & 2.06 & $1.40-3.04$ \\
\hline & $1.1-1.3$ & $139 / 95$ & 3.46 & $2.33-5.13$ \\
\hline & $1.4+$ & $145 / 89$ & 4.26 & $2.83-6.42$ \\
\hline & & $\mathrm{p}$-value for trend & \multicolumn{2}{|c|}{$<0.0001$} \\
\hline \multirow[t]{5}{*}{ Cholesterol $^{\mathrm{c}}$} & $<291$ & $59 / 175$ & 1.0 & reference \\
\hline & $292-391$ & $117 / 117$ & 2.91 & $1.95-4.33$ \\
\hline & $392-525$ & $135 / 99$ & 4.10 & $2.75-6.13$ \\
\hline & $526+$ & $153 / 81$ & 5.61 & $3.75-8.50$ \\
\hline & & $\mathrm{p}$-value for trend & \multicolumn{2}{|c|}{$<0.0001$} \\
\hline \multirow[t]{5}{*}{ Iron $^{c}$} & $<11.7$ & $93 / 141$ & 1.0 & reference \\
\hline & $11.8-14.7$ & $120 / 114$ & 1.60 & $1.10-2.34$ \\
\hline & $14.8-18.2$ & $129 / 105$ & 1.75 & $1.20-2.54$ \\
\hline & $18.3+$ & $122 / 112$ & 1.55 & $1.06-2.25$ \\
\hline & & $\mathrm{p}$-value for trend & \multicolumn{2}{|c|}{0.02} \\
\hline \multirow[t]{5}{*}{ Phosphorus $^{\mathrm{c}}$} & $<864$ & $87 / 148$ & 1.0 & reference \\
\hline & $865-1056$ & $113 / 120$ & 1.70 & $1.16-2.48$ \\
\hline & $1057-1298$ & $134 / 101$ & 2.25 & $1.52-3.31$ \\
\hline & $1299+$ & $130 / 103$ & 2.34 & $1.58-3.46$ \\
\hline & & $\mathrm{p}$-value for trend & \multicolumn{2}{|c|}{$<0.0001$} \\
\hline \multirow[t]{5}{*}{ Vitamin B12 } & $<3.9$ & $76 / 158$ & 1.0 & reference \\
\hline & $4.0-5.9$ & $98 / 136$ & 1.61 & $1.09-2.38$ \\
\hline & $6.0-7.2$ & $132 / 102$ & 2.72 & $1.84-4.08$ \\
\hline & $7.3+$ & $158 / 76$ & 4.55 & $2.03-6.82$ \\
\hline & & $\mathrm{p}$-value for trend & & .0001 \\
\hline $\mathrm{NDMA}^{\mathrm{e}, \mathrm{f}}$ & $<0.04$ & $100 / 134$ & 1.0 & reference \\
\hline & $0.05-0.08$ & $115 / 120$ & 1.22 & $0.82-1.81$ \\
\hline & $0.09-0.14$ & $124 / 109$ & 1.58 & $1.07-2.34$ \\
\hline & $0.15+$ & $125 / 109$ & 1.68 & $1.14-2.48$ \\
\hline & & $\mathrm{p}$-value for trend & & .004 \\
\hline
\end{tabular}

${ }^{\mathrm{a}}$ Multivariate odds ratios adjusted for age, residence, education, body mass index, and total energy intake; ${ }^{\mathrm{b}}$ grams/day; ${ }^{\mathrm{c}}$ miligrams/day; ${ }^{\mathrm{d}}$ micrograms/ day; ${ }^{\mathrm{e}}$ nanograms/grams; ${ }^{\mathrm{f}}$ nitrosodimethylamine 
Table 5. Odds Ratios of Advanced Prostate Cancer for Meat Nutrients Stratified by Body Mass Index ${ }^{a}$

\begin{tabular}{|c|c|c|c|c|c|}
\hline \multicolumn{6}{|c|}{ Body Mass Index } \\
\hline \multirow[t]{2}{*}{ Meat nutrient } & \multicolumn{2}{|c|}{ Low BMI } & \multicolumn{2}{|c|}{ High BMI } & \multirow[t]{2}{*}{ p-value heterogeneity ${ }^{b}$} \\
\hline & $\mathrm{OR}$ & $95 \% \mathrm{CI}$ & $\mathrm{OR}$ & $95 \% \mathrm{CI}$ & \\
\hline \multirow[t]{5}{*}{ Animal protein } & 1.0 & reference & 1.0 & reference & \\
\hline & 1.84 & $1.08-3.12$ & 1.39 & $0.79-2.43$ & \\
\hline & 2.37 & $1.38-4.08$ & 3.03 & $1.73-5.89$ & \\
\hline & 2.59 & $1.48-4.52$ & 6.79 & $3.74-12.3$ & \\
\hline & \multicolumn{2}{|c|}{$0.0004^{c}$} & \multicolumn{2}{|c|}{$<0.0001$} & 0.03 \\
\hline \multirow[t]{5}{*}{ Saturated fat } & 1.0 & reference & 1.0 & reference & \\
\hline & 1.68 & $0.98-2.90$ & 2.41 & $1.37-4.23$ & \\
\hline & 2.77 & $1.64-4.67$ & 3.84 & $2.13-6.92$ & \\
\hline & 2.49 & $1.43-4.35$ & 7.19 & $3.90-13.2$ & \\
\hline & \multicolumn{2}{|c|}{0.0002} & \multicolumn{2}{|c|}{$<0.0001$} & 0.11 \\
\hline \multirow[t]{5}{*}{ Monounsaturated fat } & 1.0 & reference & 1.0 & reference & \\
\hline & 1.46 & $0.87-2.46$ & 2.39 & $1.34-4.25$ & \\
\hline & 2.22 & $1.31-3.77$ & 4.07 & $2.26-7.33$ & \\
\hline & 2.31 & $1.33-3.99$ & 9.31 & $4.97-17.5$ & \\
\hline & \multicolumn{2}{|c|}{0.001} & \multicolumn{2}{|c|}{$<0.0001$} & 0.02 \\
\hline \multirow[t]{5}{*}{ Polyunsaturated fat } & 1.0 & reference & 1.0 & reference & \\
\hline & 3.12 & $1.80-5.41$ & 2.25 & $1.31-3.86$ & \\
\hline & 2.55 & $1.47-4.41$ & 4.10 & $2.34-7.18$ & \\
\hline & 2.08 & $1.18-3.66$ & 4.04 & $2.28-7.15$ & \\
\hline & \multicolumn{2}{|c|}{0.02} & \multicolumn{2}{|c|}{$<0.0001$} & 0.11 \\
\hline Linoleic acid & 1.0 & reference & 1.0 & reference & \\
\hline & 2.05 & $1.19-3.54$ & 1.39 & $0.83-2.32$ & \\
\hline & 1.45 & $0.86-2.46$ & 1.64 & $0.96-2.80$ & \\
\hline & 1.51 & $0.88-2.61$ & 2.25 & $1.31-3.86$ & \\
\hline & & .29 & & .003 & 0.38 \\
\hline$\alpha$-Linolenic acid & 1.0 & reference & 1.0 & reference & \\
\hline & 2.28 & $1.34-3.88$ & 1.80 & $1.02-3.19$ & \\
\hline & 3.15 & $1.82-5.45$ & 3.70 & $2.10-6.51$ & \\
\hline & 2.59 & $1.53-4.73$ & 6.43 & $3.51-11.8$ & \\
\hline & & 0002 & & .0001 & 0.07 \\
\hline Cholesterol & 1.0 & reference & 1.0 & reference & \\
\hline & 2.06 & $1.17-3.60$ & 4.38 & $2.47-7.77$ & \\
\hline & 3.35 & $1.94-5.78$ & 5.23 & $2.94-9.51$ & \\
\hline & 3.34 & $1.92-5.83$ & 11.0 & $5.85-20.7$ & \\
\hline & & .0001 & & .0001 & 0.04 \\
\hline Iron & 1.0 & reference & 1.0 & reference & \\
\hline & 2.12 & $1.26-3.57$ & 1.06 & $0.61-1.83$ & \\
\hline & 1.60 & $0.94-2.75$ & 1.78 & $1.04-3.03$ & \\
\hline & 1.50 & $0.88-2.55$ & 1.58 & $0.93-2.70$ & \\
\hline & & .25 & & .03 & 0.22 \\
\hline Phosphorus & 1.0 & reference & 1.0 & reference & \\
\hline & 1.75 & $1.01-3.04$ & 1.76 & $1.03-3.01$ & \\
\hline & 2.98 & $1.68-5.27$ & 1.93 & $1.14-3.28$ & \\
\hline & 1.97 & $1.13-3.43$ & 3.10 & $1.74-5.52$ & \\
\hline & & 008 & & 0002 & 0.12 \\
\hline Vitamin B12 & 1.0 & reference & 1.0 & reference & \\
\hline & 1.40 & $0.84-2.34$ & 1.61 & $0.89-2.89$ & \\
\hline & 1.96 & $1.14-3.35$ & 4.15 & $2.35-7.34$ & \\
\hline & 2.47 & $1.39-4.28$ & 7.95 & $4.37-14.5$ & \\
\hline & & 001 & & .0001 & 0.02 \\
\hline $\mathrm{NDMA}^{\mathrm{d}}$ & 1.0 & reference & 1.0 & reference & \\
\hline & 0.65 & $0.37-1.15$ & 2.48 & $1.39-4.42$ & \\
\hline & 1.05 & $0.62-1.79$ & 2.57 & $1.45-4.55$ & \\
\hline & 1.42 & $0.85-2.37$ & 2.16 & $1.20-3.88$ & \\
\hline & & 055 & & .03 & 0.005 \\
\hline
\end{tabular}

${ }^{a}$ Adjusted for age, residence, education, and total energy intake; ${ }^{\mathrm{b}}$ The $\mathrm{p}$-value for heterogeneity was estimated by the likelihood ratio test; ${ }^{\mathrm{c}} \mathrm{p}$-value for linear trend; ${ }^{\mathrm{d}}$ nitrosodimethylamine

1942 Asian Pacific Journal of Cancer Prevention, Vol 17, 2016 
Our study found elevated risks of prostate cancer among high consumers of total meat and red meat. We also found high risks of prostate cancer with intake of meat nutrients, namely animal protein, fatty acids, cholesterol, and NDMA.

According to several studies, obesity is a major risk factor for prostate cancer, particularly for aggressive prostate cancer (Giovannucci et al., 1997; Andersson et al., 1997; Bergström et al., 2001; Giovannucci et al., 2003; Freedland et al., 2005; Freedland et al., 2007; Pischon el al., 2008; Cao and Ma., 2011; Discaciatti et al., 2012). Higher BMI in initially cancer-free population was significantly associated with higher risk of future prostate cancer mortality (Cao \& Ma., 2011). Abdominal adiposity may be associated with an increased risk of advanced prostate cancer. This association may be stronger among individuals with lower body mass index (Pischon et al., 2008). According to the CUP of World Cancer Research Fund/American Institute for Cancer Research (2014), there is strong evidence that being overweight or obese increases the risk of advanced prostate cancer.

The results from our study support the hypothesis that red meat intake is a risk factor for prostate cancer and cooking methods could explain the effect of red meat and processed meat in relation with prostate carcinogenesis.

Previous studies have reported associations between meat consumption and prostate cancer risk. Some (Michaud et al., 2001; Koutros et al., 2008; Aune et al., 2009; Sinha et al., 2009; Deneo-Pellegrini et al., 2012), but not all of these studies (Cross et al., 2005; Alexander et al., 2010) reported positive associations between meat intake and risk of this malignancy. Previous cohort studies have yielded conflicting results with regard to red meat and prostate cancer risk, with some studies suggesting a positive association (Rodríguez el al., 2006), whereas others have found no association (Alexander et al., 2010).

Two case-control studies reported increased risk of aggressive prostate cancer among patients with high HCA intake (John et al., 2011; Joshi et al., 2012). According to the report by the World Cancer Research Fund/American Institute for Cancer Research (2007), processed meat intake has a suggestive role among those men afflicted with prostate cancer. In the present study, patients with prostate cancer showed an increased risk of $78 \%$ per 36 grams/day. Sinha et al (2009) studied the risk of advanced prostate cancer and found an increased risk of $32 \%$ per 25 grams per 1000 calories for processed meat consumption.

Shivappa et al (2015a) have suggested that chronic inflammation of the prostate could explain the deleterious effect of red meat consumption and fat intake in the etiology of this malignancy. The dietary inflammatory index has been also positively associated with prostate cancer among Jamaican men (Shivappa et al., 2015b). These case-control studies lend support to the hypothesis of chronic inflammation as a mechanism in prostate carcinogenesis. More precisely, interleukin- 6 and C-reactive protein are released from damaged cells and resulted in chronic inflammation of the prostate (Shivappa et al., 2013). Also, processed meat could promote a chronic inflammation of the prostate (Shivappa et al., 2015a).

The role of animal protein in prostate carcinogenesis has been examined in previous studies (Schuurman et al., 1999; Renehan et al., 2004; Allen et al., 2008). The effect of protein, and especially dairy protein, on IGF-I could be important, because high serum IGF-I levels have been associated with a moderately increased risk of prostate cancer in several prospective studies (Renehan et al., 2004; Allen et al., 2008). In our study, protein showed a major effect among obese patients.

The role of dietary lipids in cancer incidence still needs to be defined (World Cancer Research Fund/American Institute for Cancer Research, 2007). Since the prospective study of Le Marchand et al. (1994), fats were considered as major risk factors for prostatic carcinoma. In our study, saturated fat, monounsaturated fat, and polyunsaturated fat displayed higher risks among obese patients with prostate cancer. Moreover, monounsaturated fat showed an interaction with body mass index. It is important to mention that in our study, the polyunsaturated fat intake reflects indirectly the $\Omega-6$ polyunsaturated fatty acids (PUFA) which are present in common seed oils (e.g. sunflower, soy, corn) frequently used for salad dressing and frying purposes. Conversely, $\Omega-3$ PUFA, which are constituents of fatty fish (e.g. salmon, tuna, cod), are not frequently consumed. Uruguayan population has a very low intake of fish and the most preferred species are lean ones (e.g. hake, silverside). In addition, arachidonic acid, the active $\Omega-6$ fatty acid after conversion from linoleic acid (its precursor), enhances inflammation and facilitates progression of prostate cancer (Brown et al., 2010).

Dietary intake of long-chain $\Omega$-3 PUFA may reduce inflammation and in turn decrease risk of prostate cancer development and progression (Fradet et al., 2009; Gu et al., 2013). Although the dietary ratio $\Omega-6 / \Omega-3$ has been associated to an increase of prostate cancer risk (Williams et al., 2011), the literature has also reported no association between fish and/or $\Omega$-3 PUFA intake and prostate cancer (Chua et al., 2012; Crowe et al., 2014). An increased risk associated with high red meat intake has been found in the absence of an association with fat intake (Cross et al., 2005), therefore suggesting that the high fat content of red meat does not fully explain the association between red meat consumption and prostate cancer.

According to Platz et al (2008), cholesterol is a critical structural component of cellular membranes that serves to modulate fluidity and the interactions among many proteins, including those involved in cell survival signaling such as Akt. In a multi-site case-control study conducted in Uruguay, prostate cancer was positively associated with dietary cholesterol (De Stefani et al., 2011). Also, in the present study cholesterol was a strong risk factor. Moreover, the effect of dietary cholesterol was particularly high among obese patients.

In an EPIC study (Allen et al., 2008), protein intake, particularly that from animal protein and dairy sources, was positively associated with risk of prostate cancer. On the other hand, the study of Bidoli et al (2005) reported no association between protein intake and risk of prostate cancer. Further studies on the relationship between protein intake and prostate cancer are needed.

Data from the ProtecT study (Collin et al., 2010), when combined with results from all other studies (Weinstein 
et al., 2003; Hultdin et al., 2005; Johansson et al., 2008; Figueiredo et al., 2009; Ebbing et al., 2009), suggest that high circulating concentrations of vitamin B12 may be associated with an increased risk of prostate cancer. These findings are similar to our results for the relationship between vitamin B12 and prostate cancer. This is not a minor point, regarding the contribution of meat to vitamin B12 (Sharma et al., 2013), in particular in population having the highest meat intake in the world.

Iron overload may increase prostate cancer risk thorough stimulation of oxidative stress (Choi et al., 2008). On the other hand, calcium have been directly associated with risk of prostate cancer and its effect has been modulated by phosphorus intake in the Kesse et al. study (2006).

Finally, N-nitroso compounds, which are formed endogenously from red meat or from preservatives added to processed meats, could be related with prostatic carcinogenesis (Sinha et al., 2009). In our study, NDMA was associated with an increased risk of prostate cancer (OR 1.68, $95 \%$ 1.14-2.48).

The present study has both limitations and strengths. One limitation is the possibility of recall bias given the retrospective nature of exposure assessment. Participants in this study were mostly of low socioeconomic status, with little knowledge about the role that diet could play in prostate cancer. In addition, a high meat intake is not considered an unhealthy dietary habit in the Uruguayan population: this could have limited any possibility of recall bias, although we cannot exclude completely its chance of being present. Another possible limitation of our study could be a selection bias. Although difficult to exclude, we tried to prevent it by frequency-matched cases and controls on age and residence. Selection of hospital controls is another potential source of bias in case-control studies. However, when we repeated the analyses excluding each one of the types of controls, the results were similar. Measurement error in the assessment of diet by using a FFQ is another possible limitation of our study. Among the strengths of the study are a complete microscopic report of all cases (which was performed by pathologists with particular expertise in prostatic adenocarcinoma), and the very high response rate for both cases and controls.

In summary, our results provide further support for a positive association between meat and meat-derived nutrients and prostate cancer.

\section{References}

Alexander DD, Mink PJ, Cushing CA, et al (2010). A review and meta-analysis of prospective studies of red and processed meat intake and prostate cancer. Nutrition J,9,50.

Allen NE, Key TJ, Appleby PN, et al (2008). Animal foods, protein, calcium and prostate cancer risk: the European Prospective Investigation into Cancer Nutrition. Br J Cancer , 98, 1574-1581.

Andersson S-O, Wolk A, Bergström R, et al (1997). Body size and prostate cancer: a 20-year follow-up study among 135 006 Swedish Construction Workers. J Natl Cancer Inst, 89, 385-9.

Aune D, De Stefani E, Ronco A, et al (2009). Meat consumption and cancer risk: a case-control study in Uruguay. Asian Pac
J Cancer Prev, 10, 429-36.

Barrios E, Vassallo JA, Alonso R, et al (2010). III Atlas de incidencia de cancer en el uruguay 2002-2006. registro nacional de cancer. comisión honoraria de lucha contra el cancer. Montevideo (In Spanish).

Bergstrom A, Pisani P, Tenet V, et al (2001). Overweight as an avoidable cause of cancer in Europe. Int J Cancer, 91, 421-30.

Bidoli E, Talamini R, Bosetti C, et al (2005). Macronutrients, fatty acids, cholesterol and prostate cancer risk. Ann Oncol, 16, 152-7.

Brown MD, Hart C, Gazi E, et al (2010). Influence of omega-6 PUFA arachidonic acid and bone marrow adipocytes on metastatic spread from prostate cancer. Br J Cancer, 102, 403-13.

Cao Y, Ma J (2011). Body-mass index, prostate cancer-specific mortality and biochemical recurrence: A systematic review and meta-analysis. Cancer Prev Res, 4, 486-501.

Choi JY, Neuhouser ML, Barnett MJ, et al (2008). Iron intake, oxidative stress-related genes (Mn SOD and MPO) and prostate cancer risk in CARET cohort. Carcinogenesis, 29, 964-70.

Chua ME, Sio MCD, Sorongon MC, et al (2012). Relationship of dietary intake of omega- 3 and omega- 6 fatty acids with risk of prostate cancer development: a meta-analysis of prospective studies and review of literature. Prostate Cancer 2012, 10, 826254.

Collin SM, Metcalfe C, Refsum H, et al (2010). Circulating folate, vitamin B12, homocysteine, vitamin b12 transport proteins, and risk of prostate cancer: a case-control study, systematic review, and meta-analysis. Cancer Epidemiol Biomarkers Prev, 19, 1632-42.

Cross AJ, Peters U, Kirsh VA, et al (2005). A prospective study of meat and meat mutagens and prostate cancer risk. Cancer Res, 65, 11779-84.

Crowe FL, Appleby PN, Travis RC, et al. (2014). Circulating Fatty Acids and Prostate Cancer Risk: Individual Participant Meta-Analysis of Prospective Studies. J Natl Cancer Inst 106, 240.

Deneo-Pellegrini H, De Stefani E, Ronco A, et al (1999). Foods, nutrients and prostate cancer: a case-control study in Uruguay. Br J Cancer, 80, 591-7.

Deneo-Pellegrini H, Ronco AL, De Stefani E, et al (2012). Food groups and risk of prostate cancer: a case-control study in Uruguay. Cancer Causes Control, 23, 1031-8.

De Stefani E, Deneo-Pellegrini H, Ronco AL, et al (2011). Dietary cholesterol and risk of cancer: A multi-site casecontrol study in Uruguay. Med Hypotheses Res, 7, 17-25.

Discacciati A, Orsini N, Wolk A, et al (2012). Body mass index and incidence of localized and advanced prostate cancer-a dose-response meta-analysis of prospective studies. Ann Oncol, 23, 1665-71.

Ebbing M, Bønna KH, Nygard O, et al (2009). Cancer incidence and mortality after treatment with folic acid and vitamin B12. JAMA, 302, 2119-26.

FAO (2001). Food balance sheets. A handbook. Food and Agriculture Organization of the United Nations. Rome.

Figueiredo JC, Grau MV, Haile RW, et al (2009). Folic acid and risk of prostate cancer: results from a randomized clinical trial. J Natl Cancer Inst, 101, 432-5.

Fradet V, Cheng I, Casey G, et al (2009). Dietary Omega-3 Fatty Acids, Cyclooxygenase-2 Genetic Variation, and Aggressive Prostate Cancer Risk. Clin Cancer Res, 15, 2559.

Freedland SJ, Giovannucci E, Platz EA (2006). Are findings from studies of obesity and prostate cancer really in conflict? Cancer Causes Control, 17, 5-9.

Freedland SJ, Platz EA (2007). Obesity and prostate cancer: 
making sense out apparently conflicting data. Epidemiologic Rev, 29, 88-97.

Giovannucci E, Rimm EB, Stampfer MJ, et al (1997). Height, body weight, and risk of prostate cancer. Cancer Epidemiol Biomarkers Prev, 6, 557-563.

Giovannucci E, Rimm EB, Liu Y, et al. (2003). Body Mass Index and risk of prostate cancer in U.S. Health Professionals. $J$ Natl Cancer Inst, 95, 1240-4.

Gu Z, Suburu J, Chen H, et al (2013). Mechanisms of Omega-3 Polyunsaturated Fatty Acids in Prostate Cancer Prevention. Biomed Res Int, 2013, 10, 824563

Hosmer DW Jr, Lemeshow S (1980). Goodness-of-fit tests for the multiple logistic regression model. Comm Statistics, $\mathbf{9}$, 1043-69.

Hultdin J, Van Guelpen B, Bergh A, et al (2005). Plasma folate, vitamin B12, and homocysteine and prostate cancer risk: A prospective study. Int J Cancer, 113, 819-24.

Johansson M, Appleby PN, Allen NE, et al (2008). Circulating concentrations of folate and vitamin B12 in relation to prostate cancer risk: results from the European Prospective Investigation into Cancer and Nutrition. Cancer Epidemiol Biomarkers Prev, 17, 279-85.

John EM, Stern MC, Sinha R, et al (2011). Meat consumption, cooking practices, meat mutagens and risk of prostate cancer. Nutr Cancer, 63, 525-37.

Joshi AD, Corral R, Catsburg C, et al. (2012). Red meat and poultry, cooking practices, genetic susceptibility and risk of prostate cancer: results from a miltiethnic case-control study. Carcinogenesis, 33, 2108-2118.

Kesse E, Bertrais S, Astorg P, et al (2006). Dairy products, calcium and phosphorus intake and the risk of prostate cancer: results of the French prospective SU.VI.MAX (supplémentation en vitamines et minéraux antioxydants) study. Br J Nutr, 95, 539-45.

Koutros S, Cross AJ, Sandler DP, et al. (2008). Meat and meat mutagens and risk of prostate cancer in the Agricultural Health Study. Cancer Epidemiol Biomarkers Prev, 17, 80-7.

Kolonel LN (2001). Fat, meat and prostate cancer. Epidemiologic Rev, 23, 72-81.

Le Marchand L, Kolonel LN, Wilkens LR, et al (1994). Animal fat consumption and prostate cancer: a prospective study in Hawaii. Epidemiol, 5, 276-82.

Matos E, Brandani A (2002). Review on meat consumption and cancer in South America. Mutat Res, 506-507, 243-9.

Mazzei ME, Puchulu MR, Rochaix MA (1995). Tabla de Composición Química de Alimentos. Segunda Edicinn. CENEXA (In Spanish).

Michaud DS, Augustsson K, Rimm EB, et al (2001). A prospective study on intake of animal products and risk of prostate cancer. Cancer Causes Control, 12, 557-67.

Pischon T, Boeing H, Weikert S, et al (2008). Body size and risk of prostate cancer in the European Prospective Investigation into Cancer and Nutrition. Cancer Epidemiol Biomarkers Prev, 17, 3252-61.

Platz EA, Clinton SK, Giovannucci E (2008). Association between plasma cholesterol and prostate cancer in the PSA era. Int J Cancer, 123, 1693-8.

Punnen S, Hardin J, Chen I, et al (2011). Impact of meat consumption, preparation, and mutagens on aggresive prostate cancer. PloS One, 6, 27711.

Renehan AG, Zwahlen M, Minder C, et al (2004). Insulin-like growth factor (IGF)-I, IGF binding protein-3, and cancer risk: systematic review and meta-regression. Lancet, $\mathbf{3 6 3}$, 1346-53.

Richman EL, Kenfield SA, Stampfer MJ, et al (2011). Egg, red meat, and poultry intake and risk of lethal prostate cancer in the prostate specific antigen-era: incidence and survival.
Cancer Prev Res, 4, 2110-21.

Rodriguez C, McCullough ML, Mondul AM, et al (2006). Meat consumption among black and white men and risk of prostate cancer in the Cancer Prevention Study II Nutrition Cohort. Cancer Epidemiol Biomarkers Prev, 15, 211-6.

Ronco AL, De Stefani E, Boffetta P, et al (2006). Food patterns and risk of breast cancer: A factor analysis in Uruguay. Int J Cancer, 119, 1672-8.

Rothman KJ, Greenland S, Lash TL (2008). Modern Epidemiology. Third Edition. Lippincott Williams \& Wilkins (Philadelphia).

Schuurman AG, van den Brandt PA, Dorant E, et al (1999). Animal products, calcium and protein and prostate cancer risk in the Netherlands Cohort Study. Br J Cancer, 80, 1107-13.

Sharma S, Sheehy T, Kolonel LN (2013). Contribution of meat to vitamin B12, iron and zinc intakes in five ethnic groups in the USA: implications for developing food-based dietary guidelines. J Human Nutr Dietetics, 26, 156-68.

Shivappa N, Steck SE, Hurley TG, et al (2013). A populationbased dietary inflammatory index predicts levels of C-relative protein in the Seasonal Variation of Blood Cholesterol Study (SEASONS), Publ Health Nutr, 17, 1825-33.

Shivappa N, Bosetti C, Zuchetto A, et al (2015). Association between dietary inflammatory index and prostate cancer among Italian men. Br J Nutr, 113, 278-83.

Shivappa N, Jackson MD, Bennett F, et al (2015). Increased dietary inflammatory (DII) is associated with increased risk of prostate cancer in Jamaican men. Nutr Cancer, 67, 941-948.

StataCorp (2013). Stata Statistical Software: Release 13.1. College Station, TX: StataCorp LP.

Weistein SJ, Hartman TJ, Stolzenberg-Solomon R, et al (2003). Null association between prostate cancer and serum folate, vitamin b6, vitamin B12, and homocysteine. Cancer Epidemiol Biomarkers Prev, 12, 1271-2.

Willett W (1998). Nutritional Epidemiology. Second Edition. Monographs in Epidemiology and Biostatistics Volume 30. Oxford University Press, New York.

Williams CD, Whitley BM, Hoyo C, et al (2011). High ratio of dietary $n-6 / n-3$ polyunsaturated fatty acids is associated with increased risk of prostate cancer. Nutr Res, 31, 1-8.

World Cancer Research Fund/American Institute for Cancer Research (1997). Food, Nutrition and the Prevention of Cancer: a global perspective. Washington DC.

World Cancer Research Fund/American Institute for Cancer Research (2007). Food, Nutrition, Physical Activity, and the Prevention of Cancer: a Global Perspective. Washington DC: AICR.

World Cancer Research Fund/American Institute for Cancer Research (2014). Continuous Update Project Report: Diet, Nutrition, Physical Activity, and Prostate Cancer. 